\title{
A new approach to concordance in mid-infrared spectromicroscopy mapping of malignant tumors
}

\author{
KAISER ALI $^{1}$, TODD REICHERT ${ }^{2}$, DANIEL GOMEZ ${ }^{3}$, YANJIE LU ${ }^{1}$, \\ ALEXANDER JAN $^{4}$ and COLLEEN CHRISTENSEN ${ }^{5}$ \\ ${ }^{1}$ Division of Pediatric Hematology and Oncology, Saskatoon Cancer Centre, Cancer Research Unit, \\ Saskatchewan Cancer Agency and University of Saskatchewan; ${ }^{2}$ Department of Pathology, \\ University of Saskatchewan; ${ }^{3}$ Department of Pathology, Royal University Hospital; \\ ${ }^{4}$ Queensland University of Technology; ${ }^{5}$ Canadian Light Source Inc., \\ University of Saskatchewan, Saskatoon, Saskatchewan, Canada
}

Received January 8, 2010; Accepted March 18, 2010

DOI: $10.3892 /$ or_00000930

\begin{abstract}
Mid-infrared spectromicroscopy studies on biological tissue sections require accurate identification of tumorbearing areas in histology-stained and infrared-unstained tissue sections. Concordance was achieved as follows: paired stained and unstained thin $(5 \mu \mathrm{m})$ human brain tumor cryosections mounted on slides were scanned with a Nikon Coolscan 4000 film scanner at 4000 dpi, edited with Adobe Photoshop CS2 software, and both digital images saved. A digital tractile grid, developed in our laboratory, was overlaid onto both images. Boundaries of tumor-containing areas in stained sections were identified by light microscopy, and a digital boundary map constructed. The map was transferred onto the unstained spectromicroscopy tissue image, and finally layered onto the gridded, equisized, spectromicroscope-generated overview image prior to Fourier transform infrared spectromicroscopy. Accurate identification of tumor-bearing areas, normal brain tissue and transitional zones allowed for meaningful interpretation of respective spectral patterns in detecting subtle differences within biochemical profiles. This is the first reported method of a standardized technique for ensuring concordance in mapping of malignant tumors by mid-infrared spectromicroscopy. This technique is applicable to all biological thin tissue sections, and serves to enhance accuracy of concordance between globar- and synchrotron-light generated infrared data with that obtained by conventional light microscopy.
\end{abstract}

Correspondence to: Dr Kaiser Ali, Division of Pediatric Hematology and Oncology, Saskatoon Cancer Centre, Cancer Research Unit, Saskatchewan Cancer Agency, University of Saskatchewan, 20 Campus Drive, Saskatoon, SK S7N 4H4, Canada

E-mail: kaiser@scf.sk.ca or kaiser@saskcancer.ca

Key words: concordance, digital tractile grid, boundary demarcation, tumor tissue sections, mid-infrared spectromicroscopy

\section{Introduction}

The prognosis of children and adults diagnosed with histological high grade malignant brain tumors is poor (1). One of the main obstacles to cure in these types of tumors is the limited ability to visually determine tumor margins during surgical resection. In addition, incomplete definition of tumor boundaries by computerized tomography or magnetic resonance imaging scans in planning for treatment with radiation therapy can result in tumor progression within, or adjacent to, the radiation portals.

At the present time, light microscopy examination of hematoxylin and eosin $(\mathrm{H} \& \mathrm{E})$ stained tissue sections is the conventional method for histological diagnosis at the cellular level. In cases of brain tumors, a panel of complementary immunohistochemical stains are used for differentiating between the various types as defined by standardized classification systems, such as that developed by the World Health Organization (WHO) (2).

In the majority of brain tumor samples, light microscopy examination of slides containing H\&E stained neoplastic tissue sections reveal areas of tumor, either in the form of massive infiltration, admixed with normal cells and connective tissue, or both. However, unstained tissue sections required for mid-infrared (mid-IR) spectromicroscopy do not permit identification of any tissue components, benign or malignant, as it is not possible to identify cell types in unstained tissues (Fig. 1).

In conducting mid-IR spectromicroscopy experiments using thin tissue sections, concordant identification of normal and abnormal tissue components and their distribution on hematoxylin and eosin (H\&E) stained, and mid-IR unstained, slides is imperative. We have developed a novel technique to maximize concordance in delineating normal and malignant tissue components, and their interfaces, in tissue sections.

The long term objectives of our research studies are to identify characteristic spectral patterns of malignant human brain tumors, as compared to normal brain tissue, and of the interfacing transitional zones, by means of mid-IR spectromicroscopy. The first step in achieving these objectives 


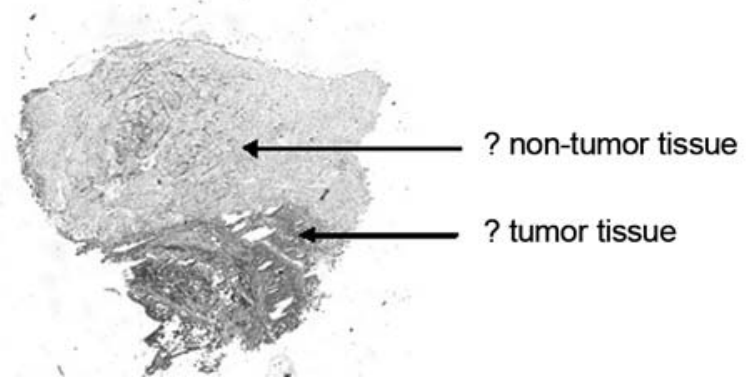

Figure 1. Unstained IR tissue section demonstrating inability to distinguish between tumor and non-tumor-bearing areas.

requires concordant identification of tumor-bearing areas in thin tissue sections for characterization by i) conventional light microscopy (H\&E stained tissue sections) and ii) midIR spectromicroscopy (unstained sections).

\section{Materials and methods}

Approval for this study and its informed consent form was obtained from the University of Saskatchewan Biomedical Research Ethics Board. Responsibility for obtaining informed consent from eligible patients in advance of their elective surgery was delegated to their respective neurosurgeons. After the first brain tissue sample was dispatched from the operating room for diagnostic studies, a second specimen was provided for research purposes. The latter specimen was snap-frozen in liquid nitrogen within $30 \mathrm{~min}$ of removal, and then cryopreserved at $-80^{\circ} \mathrm{C}$ in preparation for $\mathrm{H} \& \mathrm{E}$ staining, mid-IR spectroscopy and, as part of a correlative study, for N-myristoyl-transferase (NMT) assays (3).

Frozen tissue was placed on a cooled chuck, to which a minimum of mounting medium had been applied. Paired tissue sections $5 \mu \mathrm{m}$ in thickness were cut in a cryostat (Leica CM 1900-3-1, Germany) at $-20^{\circ} \mathrm{C}$. The first section was mounted on a glass slide for $\mathrm{H} \& \mathrm{E}$ stain, and the section immediately following was mounted on an IR-reflectance slide (Kevley Technologies, Chesterland, $\mathrm{OH}$ ) for mid-IR spectromicroscopy.

Concordance of areas to be mapped in a given brain tissue sample was achieved in the following manner: the paired stained and unstained tissue cryosections were scanned with a Nikon Coolscan 4000 film scanner at 4000 dpi to generate digital images. Boundaries around the images were cropped using an Adobe Photoshop CS2 software program. Using the same software, a 'real size' $0.5 \mathrm{~mm}^{2}$ grid map with alphanumeric coordinates covering a total surface area of $770 \mathrm{~mm}^{2}$ was developed. This grid was then superimposed as an added layer onto both images, using matched reference points (Fig. 2). Examination under light microscopy of the stained tissue section identified areas of tumor, the boundaries of which were delineated by means of a computerized contour line drawn over the gridded digital image (Fig. 3A). The same boundary demarcations were then transferred onto the gridded, unstained, IR tissue image (Fig. 3B). A third, digitized overview (OV) image, in preparation for spectroscopy of the unstained tissue section, was generated by a
A

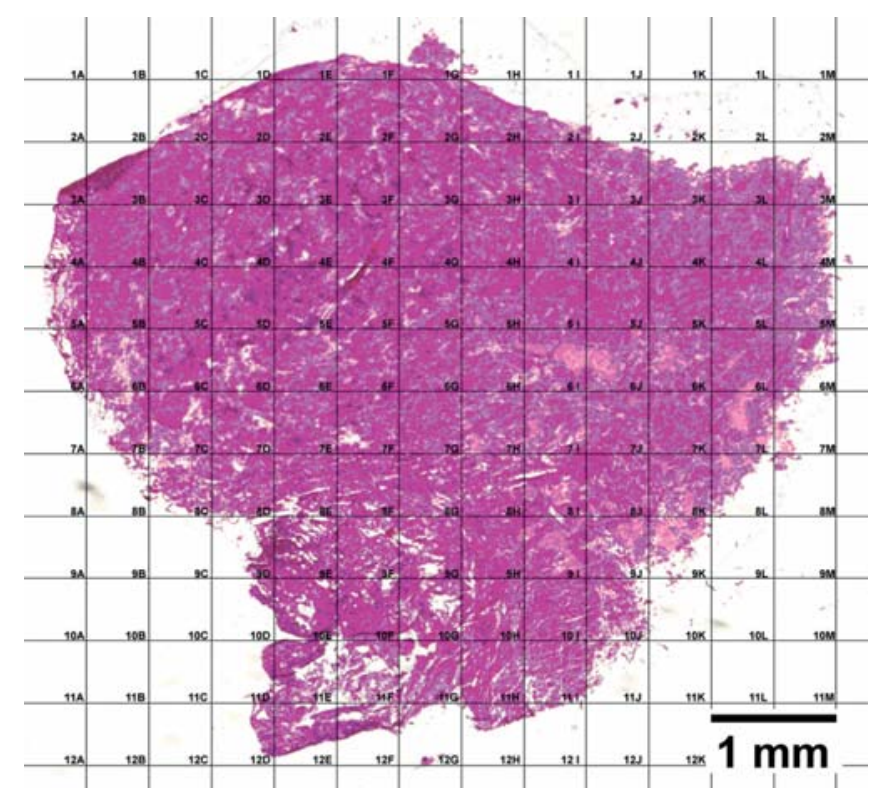

B

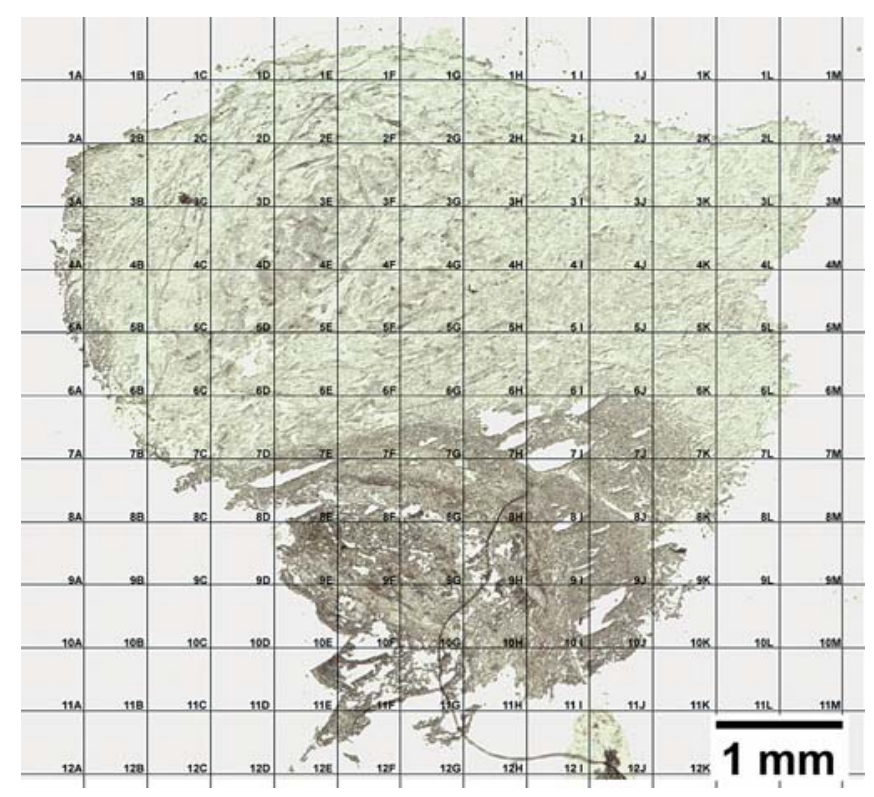

Figure 2. (A) H\&E-stained tissue section with overlaid grid. (B) IR-unstained tissue section with overlaid grid.

spectromicroscope (Hyperion IR microscope, Bruker Optics Inc., Billercia, MA) with inbuilt OPUS software.

Using the following mathematical formula, the width of all three digital images were then converted to conform to identical measurements:

Distance between diagonal points on IR image/

Distance between diagonal points on OV image/ $x$ Total width of $\mathrm{OV}$ file $=\mathrm{Z}$ (value indicating ratio of sizes)

The resolutions of all three images were equalized to 1950 ppi. Next, the tractile grid, followed by the contour boundary demarcations, were overlaid onto the OV image. Finally, the designated area for raster scanning, within the 
A

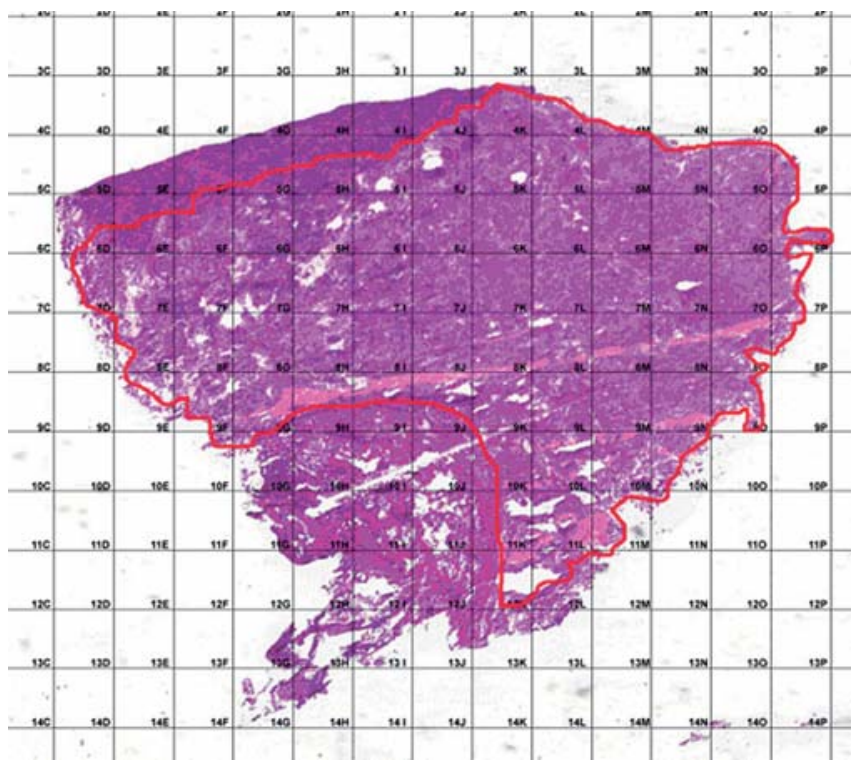

B

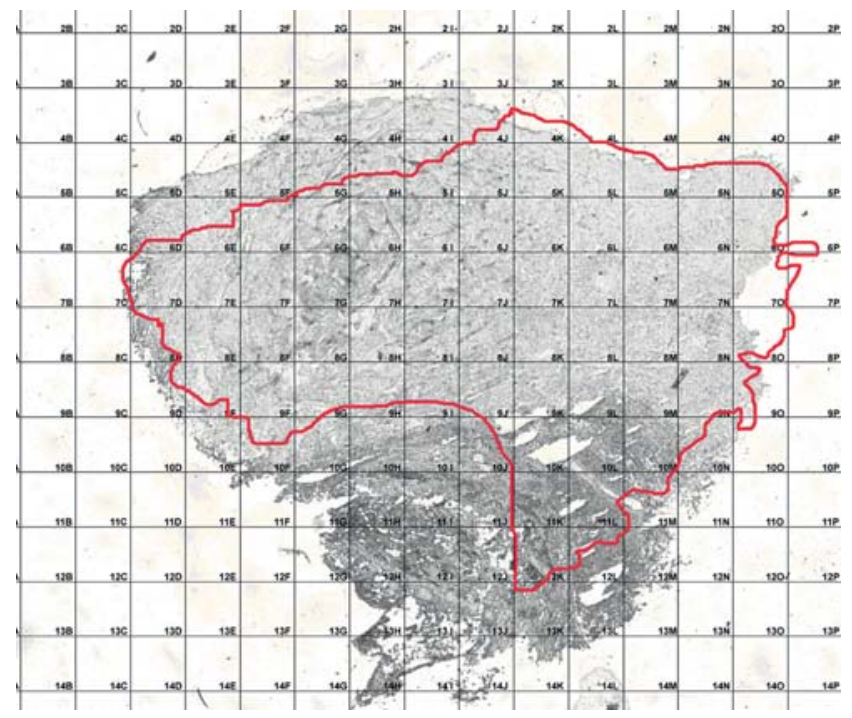

Figure 3. (A) Equisized H\&E-stained tissue section with concordant grid and tumor-bearing area demarcated within digital (red-lined) boundaries (B) Equisized paired IR-unstained tissue section with concordant grid and overlaid digital boundary map transferred from (A).

concordant tumor-bearing area on the OV image, was mapped out in preparation for Fourier transform infrared spectromicroscopy (Fig. 4).

\section{Results and Discussion}

Our method addresses two problems: i) technical: setting the boundaries for the raster map poses a challenge because the spectromicroscopy tissue section must remain unstained. Fixatives as well as chromophores in stains used in standard histological techniques significantly alter the vibrational spectral profiles of the tissue being examined (4). Our solution is to create, and work with, a high resolution digital color

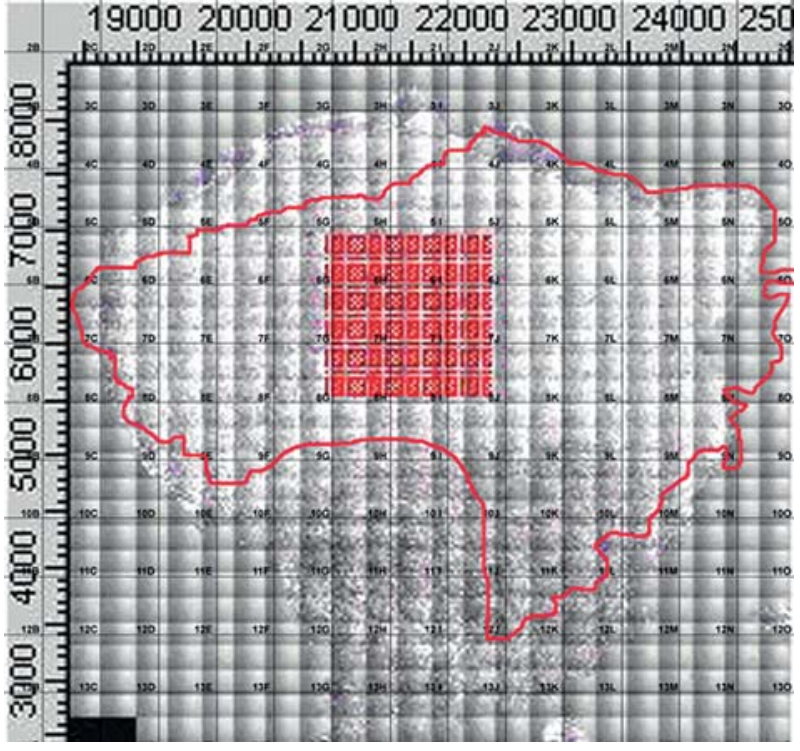

Figure 4. Equisized spectromicroscopy overview image of tissue section with raster map (red square) outlined within tumor-bearing area, in preparation for mid-IR spectroscopy (note matched grid coordinates in all images).

image of unstained tissue section with the Nikon Coolscan scanner, thereby conserving the normal biochemical composition of the tissue section for purposes of spectromicroscopical evaluation. Our technique also provides a standardized and reproducible method of ensuring that either tumor tissue, normal tissue, transitional zones inclusive of both these components, or any combination thereof are included in the raster map area for purposes of spectromicroscopical analyses. ii) Subjectivity: light microscopical determinations of tumor-bearing areas identified independently by the pathologist and the spectromicroscopist are prone to error arising from subjective interpretation. As well, repeated handling of tissue sections on glass or reflectance slides carry the risk of physical damage. These problems are resolved by the production of a working copy digital image of the H\&E stained tissue section on which a standardized digital grid is overlaid. The pathologist then clearly outlines, by means of digital linear demarcation, tumor boundaries corresponding to those detected on light microscopical examination. The final composite image serves as a template which permits the spectromicroscopist to easily identify the setup area for the raster map. The OPUS program, integrated into the mid-IR spectromicroscope, allows the OV image and raster map it generates to be saved for repeated experiments. Therefore, any number and combination of areas of normal tissue, transitional zones, and tumor on the same tissue section can be identified for repeated experiments.

The advantages of the standardized grid template are as follows: i) it standardizes sizing of all digital images, and also provides measurement ability. A tractile, 'real size' scale is built into the grid lines, so there is no need to equate the grid to the image magnification. Magnification slows down the mapping process and alters the coordinates, rendering them inaccurate. ii) Repeated digital drawing of tumor perimeters on the unstained mid-IR image is not required. The demarcation lines are directly transferable from those drawn 
on the H\&E stained digital image. iii) For purposes of identification, the pathologist digitally delineates areas of tumor onto the composite gridded image. In cases of tissue sections entirely infiltrated by tumor, this step may be eliminated. The grid can therefore serve both as a standardized tool as well as an assessment tool. In the absence of a pathologist, the grid can be used as anchor points, thereby maintaining consistency.

To our knowledge, this is the first reported method of its kind for confirming concordance of tumor and non-tumor containing areas on H\&E-stained and mid-IR spectromicroscopy-unstained thin tissue sections. This standardized technique is applicable to all biological tissue sections, and will enhance accuracy of data extraction and interpretation in research into spectroscopy using both globar- and synchrotrongenerated light sources.

This is the first reported method of a standardized technique for concordant mid-IR spectromicroscopy mapping of tumorbearing tissue sections. It allows: i) concordant identification of tumor tissue within paired histology-stained and mid-IR spectromicroscopy-unstained tissue sections and, ii) precise mapping of tumor-bearing, non-tumor bearing, and transitional zones for raster scanning by mid-IR spectromicroscopy. This methodology is applicable to all biological thin tissue sections requiring mid-IR spectromicroscopic analyses, and will enhance accuracy of concordant data interpretation in globar- and synchrotron-light generated infrared data with that obtained by means of conventional histological techniques.

\section{Acknowledgements}

The research described in this manuscript was performed at the Canadian Light Source, which is supported by NSERC, NRC, CIHR and the University of Saskatchewan. We thank the following persons for their contributions: Tim May, M.Sc., Staff Scientist, Canadian Light Source, Inc., for continuing support and advice, Shannon Klassen, A.R.T., Department of Pathology, Saskatoon City Hospital, for preparation and staining of tissue sections, and Ylona Loessl and Jennifer Hart, Department of Pediatric Oncology, Saskatoon Cancer Centre, for secretarial assistance. This study was funded in part by grants from the following sources: i) Saskatchewan Synchrotron Institute and ii) Office of the Vice-President, Research, College of Medicine, University of Saskatchewan.

\section{References}

1. Kaatsch P, Rickert CH, Kühl J, Schüz J and Michaelis J: Population-based epidemiologic data on brain tumors in German children. Cancer 92: 3155-3164, 2001.

2. Kleihues P, Burger PC and Scheithauer BW: The new WHO classification of brain tumours (Review). Brain Pathol 3: 255-268, 1993.

3. Lu Y, Selvakumar P, Ali K, Shrivastav A, Bajaj G, Resch L, Griebel R, Fourney D, Meguro K and Sharma RK: Expression of N-myristoyltransferase in human brain tumors. Neurochem Res 30: 9-13, 2005

4. Jackson M, Choo LP, Watson PH, Halliday WC and Mantsch HH: Beware of connective tissue proteins: assignment and implications of collagen absorptions in infrared spectra of human tissues. Biochim Biophys Acta 1270: 1-6, 1995. 